\title{
Cascading detection model for prediction of apnea-hypopnea events based on nasal flow and arterial blood oxygen saturation
}

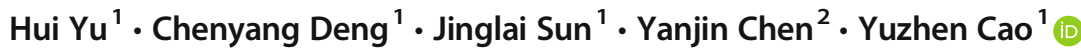 \\ Received: 29 January 2019 / Revised: 20 June 2019 / Accepted: 27 June 2019 / Published online: 5 July 2019 \\ (C) The Author(s) 2019
}

\begin{abstract}
Purpose Sleep apnea and hypopnea syndrome (SAHS) seriously affects sleep quality. In recent years, much research has focused on the detection of SAHS using various physiological signals and algorithms. The purpose of this study is to find an efficient model for detection of apnea-hypopnea events based on nasal flow and $\mathrm{SpO}_{2}$ signals.

Methods A 60-s detector and a 10-s detector were cascaded for precise detection of apnea-hypopnea (AH) events. Random forests were adopted for classification of data segments based on morphological features extracted from nasal flow and arterial blood oxygen saturation $\left(\mathrm{SpO}_{2}\right)$. Then the segments' classification results were fed into an event detector to locate the start and end time of every $\mathrm{AH}$ event and predict the $\mathrm{AH}$ index (AHI).

Results A retrospective study of 24 subjects' polysomnography recordings was conducted. According to segment analysis, the cascading detection model reached an accuracy of $88.3 \%$. While Pearson's correlation coefficient between estimated AHI and reference AHI was 0.99 , in the diagnosis of SAHS severity, the proposed method exhibited a performance with Cohen's kappa coefficient of 0.76 .

Conclusions The cascading detection model is able to detect $\mathrm{AH}$ events and provide an estimate of AHI. The results indicate that it has the potential to be a useful tool for SAHS diagnosis.
\end{abstract}

Keywords Sleep apnea and hypopnea syndrome $\cdot$ Apnea-hypopnea index · Polysomnography $\cdot$ Cascading detection model . Apnea-hypopnea events

\section{Introduction}

Sleep apnea and hypopnea syndrome (SAHS) is a prevalent sleep breathing disorder in middle-aged people. The gold standard for diagnosis of SAHS is to perform polysomnography (PSG) in a laboratory. However, PSG requires patients to sleep with many sensors for at least one night; the scoring of apneahypopnea $(\mathrm{AH})$ events can take a long time. Therefore, many researchers hope to simplify or replace PSG by using a limited number of physiological signals. Electrocardiogram (ECG) was first studied for this purpose. McNames et al. [1] found that heart rate, S-pulse amplitude, and pulse energy were correlated with SAHS. Bsoul et al. [2] cut the ECG into 60-s segments and used

Yuzhen Cao

yuzhencao18@126.com

1 Department of Biomedical Engineering, Tianjin University, Tianjin, China

2 Tianjin Hospital of ITCWM Nankai Hospital, Tianjin, China a support vector machine (SVM) for real-time detection of apnea. However, many other diseases except SAHS also affect ECG. Hence, nasal flow (NF) [3-6], arterial blood oxygen saturation $\left(\mathrm{SpO}_{2}\right)$ [7], snoring [8], or a combination of these signals [9, 10] have been adopted more recently. Gutierrez et al. [4] used the overall features of NF for the diagnosis of SAHS severity. Xie et al. [10] utilized a combination of classifiers to achieve realtime detection of SAHS based on $\mathrm{ECG}$ and $\mathrm{SpO}_{2}$. All the above studies can be roughly divided into two categories: those that predict the AH index (AHI) based on the detection of $\mathrm{AH}$ events $[2,3,5,7,9-11]$, and those that predict AHI based on the overall signal features $[1,4,6,8,12,13]$. The latter approach cannot provide time information for each $\mathrm{AH}$ event, whereas most studies in the former $[2,7,10,11]$ only involve a 60 -s segment identification which may not be accurate for predicting the segments containing multiple $\mathrm{AH}$ events and may lead to errors in the estimation of AHI. On the other hand, the methods mentioned above include rule-based [5, 7, 9], SVM [2, 10, 11], and supervised neural network $[3,11]$, which require a large number of hyperparameters to be set by experience. Therefore, we 
utilized random forests composed of classification and regression trees (CARTs) based on morphological features extracted from $\mathrm{NF}$ and $\mathrm{SpO}_{2}$ for $\mathrm{AH}$ events detection. A 60-s detector and a 10-s detector were cascaded for more precise detection of $\mathrm{AH}$ events.

\section{Materials and methods}

\section{Subjects}

The St. Vincent University Hospital/Dublin University College Sleep Apnea Syndrome Database (UCDDB) [14] public on Physionet [15] was used for a retrospective data analysis throughout this paper. The database contains 25 subjects' PSG data, including EEG, electrooculogram, submental electromyography, NF, ribcage and abdomen movements, $\mathrm{SpO}_{2}$, snoring, and body position. All signals were obtained using a Jaeger-Toennies system. The annotation files consisted of onset time and duration of respiratory events provided by an experienced specialist. The cutoff values for AHI were commonly set to 5,15 , or 30 events/h $[3,4,7,16,17]$. There were data for two non-SAHS subjects, twelve mild-SAHS subjects, five moderate-SAHS subjects, and six severe-SAHS subjects in the database. While there was a severe distortion in the NF signal of subject ucddb005 thus this recording was excluded. Consequently, totally 24 subjects' polysomnography recordings were taken into this study. The sleep-related parameters of the subjects are summarized in Table 1.

According to the American Academy of Sleep Medicine (AASM) manual [16], apnea is scored when there is a more than $90 \%$ drop in the peak signal of the pre-event baseline for $\mathrm{NF}$ with a duration longer than $10 \mathrm{~s}$. Hypopnea is scored by the following rules: (1) there is a more than $30 \%$ drop in the peak signal of the pre-event baseline for nasal pressure with a duration longer than $10 \mathrm{~s}$, accompanied by (2) more than $3 \%$ arterial oxygen desaturation or an arousal. As a result, we selected NF and $\mathrm{SpO}_{2}$ for SAHS detection. The NF signal was recorded by a thermistor while $\mathrm{SpO}_{2}$ was recorded by a finger pulse oximeter and the sampling rate of both was $8 \mathrm{~Hz}$.

\section{Study design}

The cascading detection model based on AH event detection is shown in Fig. 1. It comprises the following main steps: (1) removal of invalid data, NF signal filtering, segmentation with a sliding window, and $\mathrm{SpO}_{2}$ alignment; (2) extraction of a specific feature set from each segment; 3 ) the cascading detection model predicts each segment and outputs a sequence of segments' results; (4) the event detector corrects the invalid results in the sequence and calculates the AHI.

\section{Signal preprocessing}

Signal preprocessing comprises the following four steps: (1) removal of invalid data. Any $\mathrm{SpO}_{2}$ values lower than $50 \%$ were considered to be artifacts and removed from the analysis (5.6\% of the data). (2) NF signal filtering - a four-point sliding average filter and a third-order Butterworth high-pass filter with a cutoff frequency of $0.05 \mathrm{~Hz}$ were used to prevent highfrequency noise caused by artifacts and baseline drift in NF signal. (3) Segmentation - the original signals were segmented using a 60-s window and a 10-s window, respectively. In both cases, the step was set to $1 \mathrm{~s}$. All segments were categorized into two classes: $\mathrm{AH}$ and $\mathrm{N}$ according to the annotations. The segments containing more than $5 \mathrm{~s}$ of $\mathrm{AH}$ events were labeled as class AH. Other cases were labeled as class N. (4) $\mathrm{SpO}_{2}$ alignment. As $\mathrm{SpO}_{2}$ responds slowly to AH events [18], a time advance of $\tau \mathrm{s}(0<\tau<30)$ was applied in $\mathrm{SpO}_{2}$. The results showed that the model performed best with $\tau$ set to 23 s. After preprocessing, the number of extracted segments were 487,974 (AH, $N=44,476: 443,498)$.

\section{Feature extraction}

NF feature set According to the AASM definition of $\mathrm{AH}$ events, the amplitude of NF provides important information. Therefore, we first extracted the maximum and minimum points from each NF segment. Then, the tidal volume per breath $F t$ was calculated as the difference between two adjacent extreme points. The mean, standard deviation, and range of the tidal volume ( $F$ mean, Fstd, Fran) were extracted within each segment. Besides, we calculated the maximum value of the tidal volume every $30 \mathrm{~s}$ using Eq. (1):

$F b_{i}=\max \left\{F t_{i-30}, F t_{i-29}, \ldots, F t_{i-1}\right\}$

where $F t_{i}$ represents the tidal volume values in the $i$ th segment. $F b_{i}$ represents the maximum tidal volume value in $30 \mathrm{~s}$ before the $i$ th segment. The number of breaths with tidal volume drops by more than $30 \%, 70 \%$ from $F b$ were calculated within each
Table 1 Summary of sleeprelated parameters (mean \pm standard deviation)

\begin{tabular}{lllll}
\hline & Non-SAHS & Mild SAHS & Moderate SAHS & Severe SAHS \\
\hline Number of patients & 2 & 12 & 5 & 5 \\
Age (years) & $52.0 \pm 15.6$ & $48.6 \pm 8.5$ & $56.8 \pm 6.4$ & $46.6 \pm 5.5$ \\
AHI (events/h) & $4.1 \pm 5.7$ & $9.9 \pm 2.9$ & $24.6 \pm 3.9$ & $43.8 \pm 16.3$ \\
Epworth Sleepiness Score & $7.0 \pm 8.5$ & $11.6 \pm 5.1$ & $11.2 \pm 6.9$ & $12.4 \pm 7.9$ \\
\hline
\end{tabular}




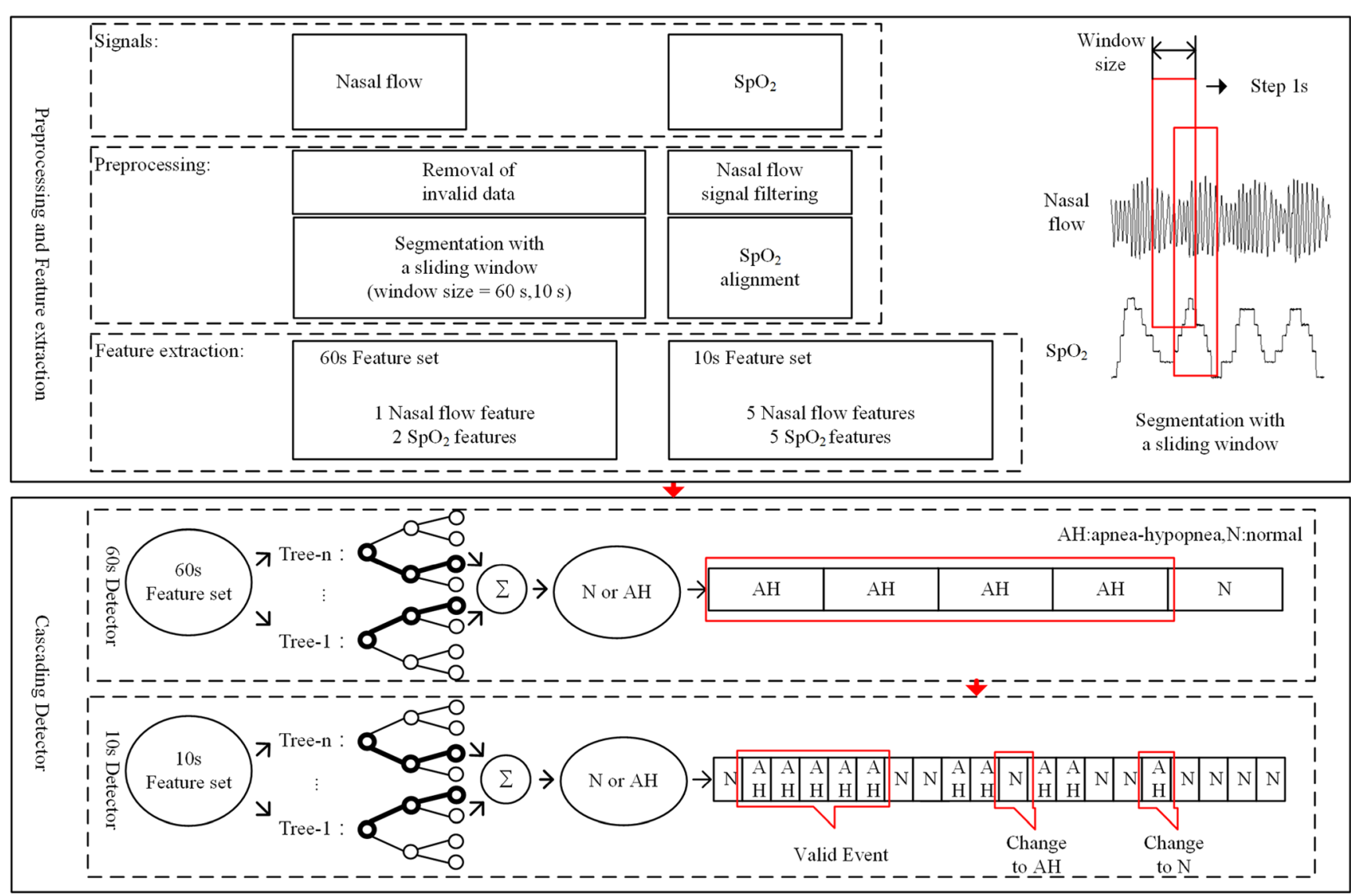

Fig. 1 Design of cascading detection model based on AH event detection

segment and denoted as Fha, Fap. And the number of breaths with tidal volume above $85 \%$ of $F b$ was also calculated and denoted as Fnor. In addition, the ratios of them to the total number of breaths (Fhap, Fapp, Fnorp) within each segment were calculated. Besides, owing to the cessation of breathing, there will be fluctuations in the breathing rate during $\mathrm{AH}$ events. One normal breath lasts for 3-5 s; energy will be concentrated with a peak in the corresponding frequency. As a result, we took the fourth statistical moment (Fkur) in $0.2-0.4 \mathrm{~Hz}$ of NF's frequency spectrum as another feature.
$\mathrm{SpO}_{2}$ feature set We first calculated the standard deviation and range coefficients of $\mathrm{SpO}_{2}$ (Spstd, Spran) in each segment. The tendency of $\mathrm{SpO}_{2}$ in each segment (Spten) was also calculated by using the last $\mathrm{SpO}_{2}$ value minus the first $\mathrm{SpO}_{2}$ value. The commonly used feature: time $\mathrm{SpO}_{2}$ stays below 90\% [19, 20] was referred while we calculated the indices $S p 92, S p 91$ with thresholds set to $92 \%$ and $91 \%$. Besides, the maximum $\mathrm{SpO}_{2}$ value $(\mathrm{Spbm})$ and average $\mathrm{SpO}_{2}$ value (Spba) in every 30 s were computed. Then within each segment, the time $\mathrm{SpO}_{2}$ stays below $98 \%$ of Spbm and that below $98 \%$ of Spba were calculated and

Table 2 Features and their definitions

\begin{tabular}{cll}
\hline Index & Name & Definition \\
\hline 1 & Fmean, Fst, Fran & Average, standard deviation, and range of tidal volume \\
2 & Fha, Fhap & Number of breaths with a reduction more than $30 \%$ in tidal volume and its ratio to total number of breaths \\
3 & Fap, Fapp & Number of breaths with a reduction more than $70 \%$ in tidal volume and its ratio to total number of breaths \\
4 & Fnor, Fnorp & Number of breaths with a reduction less than $15 \%$ in tidal volume and its ratio to total number of breaths \\
5 & Fkur & Fourth statistical moment in $0.2-0.4 \mathrm{~Hz}$ of NF's frequency spectrum \\
6 & Spstd, Spran & Standard deviation and range of $\mathrm{SpO}_{2}$ \\
7 & Spten & Tendency of $\mathrm{SpO}_{2}$ \\
8 & Spdum, Spdua & Duration of $\mathrm{SpO}_{2}$ desaturation \\
9 & Spldm, Splda & Level of $\mathrm{SpO}_{2} \mathrm{desaturation}^{-}$ \\
10 & Sp92, Sp91 & Duration of $\mathrm{SpO}_{2}$ staying below 92 and $91 \%$ \\
\hline
\end{tabular}


Table 3 Results for segments

\begin{tabular}{|c|c|c|c|c|c|c|}
\hline & & \multicolumn{2}{|c|}{ Reference } & \multirow[t]{2}{*}{$\operatorname{ACC}(\%)$} & \multirow[t]{2}{*}{ SEN (\%) } & \multirow[t]{2}{*}{ SPE (\%) } \\
\hline & & $\mathrm{AH}$ & $N$ & & & \\
\hline \multirow[t]{2}{*}{ Estimated } & $\mathrm{AH}$ & 33,429 & 46,247 & 88.3 & 75.2 & 89.6 \\
\hline & $\mathrm{N}$ & 11,047 & 397,251 & & & \\
\hline
\end{tabular}

$A C C$ accuracy, SEN sensitivity, SPE specificity

denoted as Spdum, Spdua respectively. Finally, the level of oxygen desaturation Spldm and Splda in each segment was calculated as Eqs. (2) and (3) show.

$\operatorname{Spldm}_{i}=\operatorname{Spbm}_{i}-\operatorname{mean}\left\{\mathrm{Sp}_{i}\right\}$

$\operatorname{Splda}_{i}=S p b a_{i}-\operatorname{mean}\left\{S p_{i}\right\}$

where $i$ represents the $i$ th segment. $S p_{i}$ represents the $\mathrm{SpO}_{2}$ values in the $i$ th segment. $S_{p b m}, S p b a_{i}$ represent the maximum and average $\mathrm{SpO}_{2}$ value in $30 \mathrm{~s}$ before the $i$ th segment respectively. The total feature set is shown in Table 2 .

\section{Design of cascading detector}

The cascading detector contained two parts. The first was a random forest consisting of 10 CARTs for the prediction of $60 \mathrm{~s}$ segments. This could screen out most of the $\mathrm{N}$ segments while retaining the $\mathrm{AH}$ segments. The second part was a random forest consisting of 20 CARTs for the prediction of $10 \mathrm{~s}$
Fig. 2 a $\mathrm{AH}$ event estimation result for mild-SAHS. b AH event estimation result for mediumSAHS. c AH event estimation result for severe-SAHS
Reference Events

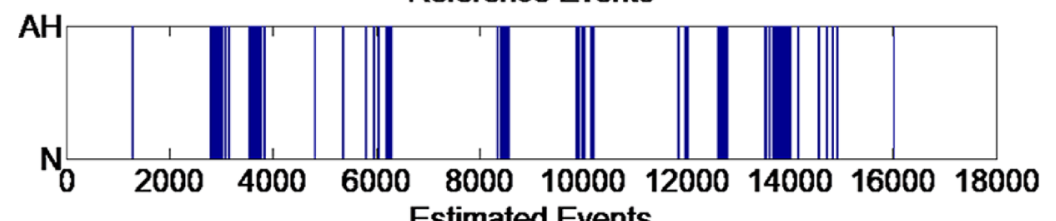

a

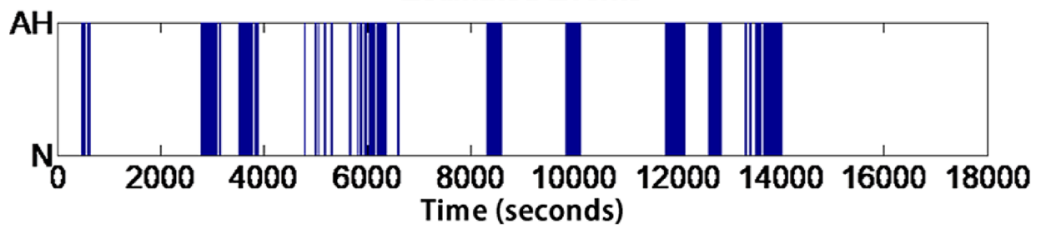

Reference Events

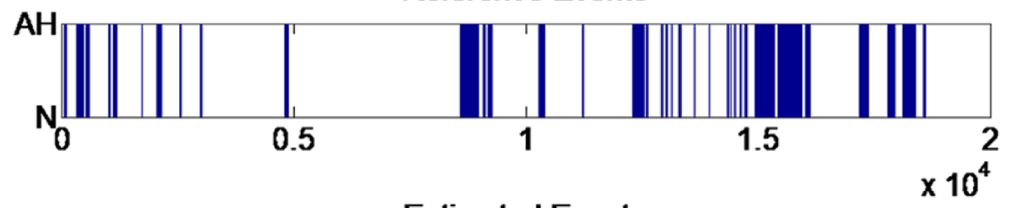

b

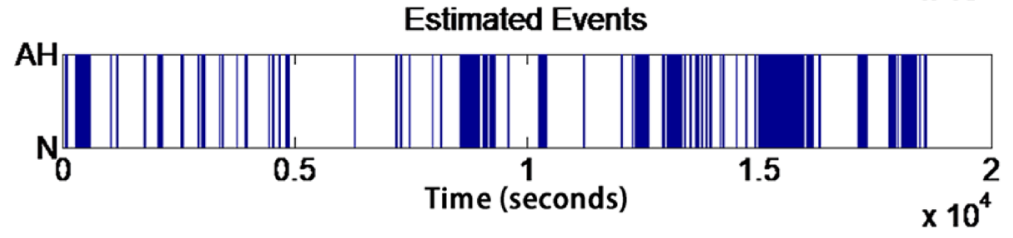

Reference Events

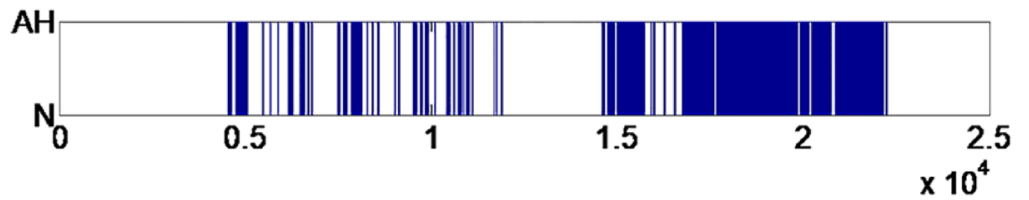

Estimated Events

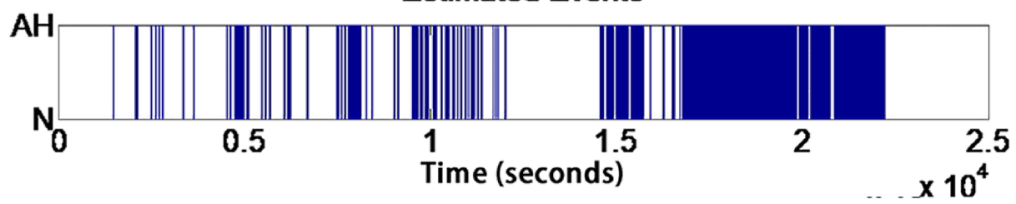



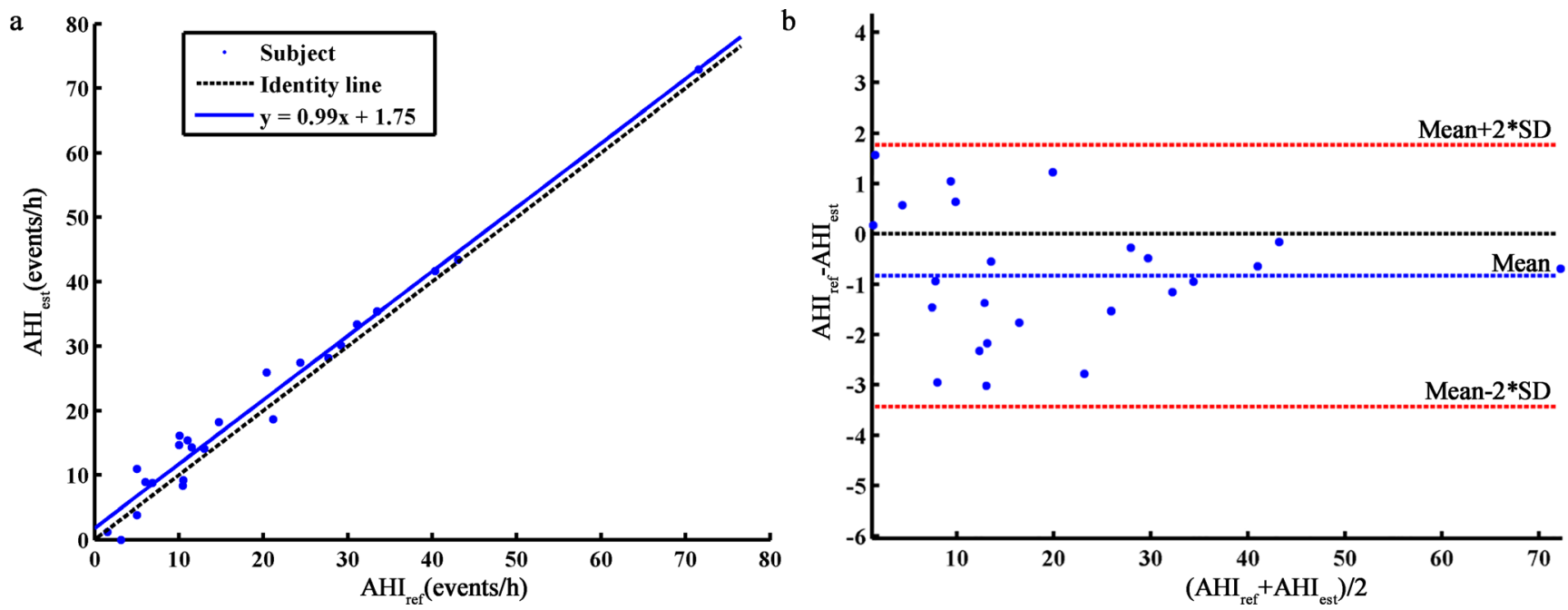

Fig. 3 a Scatter plot of $\mathrm{AHI}_{\text {est }}$ and $\mathrm{AHI}_{\text {ref. }}$ b Bland-Altman plot of $\mathrm{AHI}_{\text {est }}$ and $\mathrm{AHI}_{\text {ref }}$

segments. Based on the results of the 60-s detector, the 10 -s detector was able to detect $\mathrm{AH}$ events more precisely.

Note that the $60 \mathrm{~s}$ detector was trained using a feature set composed of features 2, 6, and 8 in Table 2 in order to improve the training speed. The results indicated that there was almost no effect on the performance. Owing to the imbalance in the number of $\mathrm{AH}$ and $\mathrm{N}$ segments, the weights for the two classes in CARTs were set to inverse ratio of their numbers.

A twofold cross-validation was used in the test. Each time, half of the segments were used for training with the remaining half used for testing. The cascading detector output the sequence composed of the prediction results of the $10 \mathrm{~s} \mathrm{seg-}$ ments. The detector was trained on a computer with an i57600k CPU and 8 G RAM.

\section{Design of event detector}

The sequence predicted by the cascading detector was then fed into the event detector to correct invalid results following two rules; (1) Only more than 10 consecutive segments classified as $\mathrm{AH}$ were considered to be one valid $\mathrm{AH}$ event. As the original data were segmented by a 10 -s window, and one $\mathrm{AH}$ event lasts at least $10 \mathrm{~s}$, so one $\mathrm{AH}$ event corresponded to at least 10 consecutive AH segments. Any segment which did not meet the rule was modified to class N. (2) The number of segments classified as $\mathrm{N}$ between two valid $\mathrm{AH}$ segments was supposed to be more than five. This was also determined by the way of data segmentation. Any segment that did not meet the rule was reset to class $\mathrm{AH}$.

\section{Results}

The cascading detection model was able to estimate $\mathrm{AHI}$ and provide the time information for each $\mathrm{AH}$ event. We analyzed its performance with respect to two aspects: segments and AHI.

\section{Segment analysis}

The prediction results for the segment-by-segment analysis are shown in Table 3 . The cascading detection model achieved an accuracy of $88.3 \%$, a sensitivity of $75.2 \%$, and a specificity of $89.6 \%$ for 487,974 test segments.

Figure 2 displays the AH event estimation results for one mild-SAHS, medium-SAHS, and severe-SAHS subject. For the mild-SAHS subject, the accuracy, sensitivity and specificity were $92.9 \%, 80.6 \%$, and $94.0 \%$. The corresponding values for the medium-SAHS subject were $92.2 \%, 82.2 \%$, and $93.6 \%$ while $86.7 \%, 81.1 \%$, and $88.2 \%$ for the severe one.
Table 4 Comparison of number of $\mathrm{AH}$ events, duration of $\mathrm{AH}$ events, and AHI predicted by cascading model with PSG (mean \pm standard deviation)

\begin{tabular}{|c|c|c|c|c|c|c|}
\hline \multirow[t]{2}{*}{ Group } & \multicolumn{2}{|c|}{ Number of AH events } & \multicolumn{2}{|c|}{ Duration of AH events (min) } & \multicolumn{2}{|c|}{ AHI (events/h) } \\
\hline & Reference & Estimated & Reference & Estimated & Reference & Estimated \\
\hline Non & $17.0 \pm 7.0$ & $9.0 \pm 8.3$ & $4.6 \pm 2.3$ & $6.2 \pm 4.6$ & $3.2 \pm 1.4$ & $1.7 \pm 1.6$ \\
\hline Mild & $61.5 \pm 19.4$ & $83.0 \pm 37.2$ & $18.8 \pm 6.2$ & $29.6 \pm 7.9$ & $9.9 \pm 2.8$ & $12.1 \pm 4.2$ \\
\hline Moderate & $132.2 \pm 18.7$ & $155.0 \pm 23.6$ & $42.5 \pm 14.2$ & $54.3 \pm 15.5$ & $24.6 \pm 3.5$ & $27.5 \pm 5.4$ \\
\hline Severe & $193.2 \pm 63.2$ & $200.2 \pm 63.8$ & $61.3 \pm 21.2$ & $72.2 \pm 30.8$ & $43.9 \pm 14.5$ & $45.3 \pm 14.3$ \\
\hline
\end{tabular}


Table 5 SAHS severity classification and diagnostic performance

\begin{tabular}{|c|c|c|c|c|c|c|c|c|c|c|}
\hline & & \multicolumn{4}{|c|}{ Determined from PSG } & & \multicolumn{4}{|c|}{ AHI cutoff $(\backslash)$} \\
\hline & & Non & Mild & Moderate & Severe & & $\geq 5$ & $\geq 15$ & $\geq 30$ & AVE \\
\hline \multirow[t]{4}{*}{ Estimated } & Non & 2 & 0 & 0 & 0 & SEN (\%) & 100.0 & 100.0 & 100.0 & 100.0 \\
\hline & Mild & 0 & 9 & 0 & 0 & SPE $(\%)$ & 100.0 & 78.6 & 94.7 & 91.1 \\
\hline & Moderate & 0 & 3 & 4 & 0 & PPV (\%) & 100.0 & 76.9 & 83.3 & 86.7 \\
\hline & Severe & 0 & 0 & 1 & 5 & $\operatorname{ACC}(\%)$ & 100.0 & 87.5 & 95.8 & 94.4 \\
\hline
\end{tabular}

$A C C$ accuracy, $S E N$ sensitivity, $S P E$ specificity, $P P V$ positive predictive value

\section{AHI analysis}

Figure 3(a) shows a scatter plot of the $\mathrm{AHI}\left(\mathrm{AHI}_{\text {est }}\right)$ estimated by the model and the AHI ( $\left.\mathrm{AHI}_{\mathrm{ref}}\right)$ determined from PSG. The solid line fitted shows a high correlation (Pearson's correlation coefficient $0.99, p<0.01)$ between $\mathrm{AHI}_{\mathrm{est}}$ and $\mathrm{AHI}_{\text {ref. }}$. Figure 3(b) shows the Bland-Altman plot of $\mathrm{AHI}_{\text {est }}$ and $\mathrm{AHI}_{\text {ref }}$. The average error of $\mathrm{AHI}_{\text {est }}$ and $\mathrm{AHI}_{\text {ref }}$ was -0.8 events/h, and the error range was -3.4 to 1.8 events/h (95\% confidence interval).

Table 4 compares the number of AH events, duration of $\mathrm{AH}$ events and AHI values for the cascading model and scored by PSG. Table 5 summarizes the classification results for SAHS severity. The mean values for sensitivity, specificity, PPV, and accuracy were $100.0 \%, 91.1 \%, 86.7 \%$, and $94.4 \%$, respectively, for AHI thresholds of 5, 15, and 30 events/h. Besides, the kappa coefficient for diagnosis of SAHS severity was 0.76 .

\section{Discussion}

We proposed a cascading detection model that could predict $\mathrm{AHI}$ based on AH event detection. Compared with PSG, only NF and
$\mathrm{SpO}_{2}$ were used. Previously, the original signals were commonly cut into $60 \mathrm{~s}$ segments for $\mathrm{AH}$ event detection $[2,7,10,11]$. However, the detection of AH events may not be precise based on $60 \mathrm{~s}$ segment analysis because it can only determine whether there was $\mathrm{AH}$ in the segment, while, may make mistakes for the segments containing multiple $\mathrm{AH}$ events and lead to an error in AHI estimation. Therefore, some researchers [3,9] cut the signals into shorter segments for detection. However, it is difficult to extract effective features from a segment shorter than $10 \mathrm{~s}$, because there will be no more than five complete breaths in one segment in most cases. As a result, we proposed a cascading detection model composed of a 60 -s detector and a 10 -s detector to predict $\mathrm{AH}$ events precisely. Table 3 shows the classification results for the segments. Notably, the model tended to make false positive errors. In approximately $12.1 \%$ of these errors, the amplitude of NF signal decreased by more than $30 \%$ from previous event baseline accompanied with a $\mathrm{SpO}_{2}$ desaturation, however no arousal or nasal pressure signals were adopted for identifying hypopneas. Therefore, these segments may be mistaken for class AH.

As illustrated in Fig. 3, $\mathrm{AHI}_{\mathrm{est}}$ showed high correlation with $\mathrm{AHI}_{\text {ref }}$ (Pearson correlation coefficient 0.99, $p<0.01$ ).

Table 6 Comparison with other studies

\begin{tabular}{|c|c|c|c|c|c|c|}
\hline Related work & Method & Signal & AHI cutoff & $\operatorname{ACC}(\%)$ & SEN $(\%)$ & SPE $(\%)$ \\
\hline \multirow[t]{3}{*}{ Choi et al. [3] } & \multirow[t]{3}{*}{ Convolutional neural networks } & \multirow[t]{3}{*}{ Nasal pressure } & 5 & 96.2 & 100.0 & 84.6 \\
\hline & & & 15 & 92.3 & 98.1 & 86.5 \\
\hline & & & 30 & 96.2 & 96.2 & 96.2 \\
\hline \multirow[t]{3}{*}{ Gonzalo et al. [4] } & \multirow[t]{3}{*}{ AdaBoost-Linear discriminant analysis } & \multirow[t]{3}{*}{ Nasal flow } & 5 & 86.5 & 87.1 & 80.0 \\
\hline & & & 15 & 81.0 & 85.9 & 72.9 \\
\hline & & & 30 & 82.5 & 74.2 & 90.6 \\
\hline \multirow[t]{4}{*}{ Da Woon Jung et al. [7] } & \multirow[t]{4}{*}{ Rule-based } & \multirow[t]{4}{*}{$\mathrm{SpO}_{2}$} & 5 & 97.8 & 98.6 & 94.4 \\
\hline & & & 10 & 96.7 & 98.4 & 92.9 \\
\hline & & & 15 & 95.7 & 96.4 & 94.6 \\
\hline & & & 30 & 96.7 & 97.1 & 96.5 \\
\hline \multirow[t]{3}{*}{ Our study } & \multirow[t]{3}{*}{ Cascade of random forests } & \multirow[t]{3}{*}{ Nasal flow and $\mathrm{SpO}_{2}$} & 5 & 100.0 & 100.0 & 100.0 \\
\hline & & & 15 & 87.5 & 100.0 & 78.6 \\
\hline & & & 30 & 95.8 & 100.0 & 94.7 \\
\hline
\end{tabular}

$A C C$ accuracy, SEN sensitivity, SPE specificity 
The performance of the model also showed good consistency among different subjects. On the other hand, $\mathrm{AHI}_{\text {est }}$ was slightly higher than $\mathrm{AHI}_{\text {ref. }}$ Consequently, SAHS severity was overestimated for four subjects; for the remaining 20 subjects, the model gave the correct prediction (Table 5). The kappa coefficient of the cascading detection model for diagnosis of SAHS severity was 0.76 , indicating that this method represents a powerful screening tool for SAHS.

We also tested the speed of the cascading detection model. Training required $24.7 \mathrm{~s}$, while only $20.3 \mathrm{~s}$ was needed to provide results for all segments and to predict AHI for all 24 subjects. It took $41.6 \mu$ s to predict one segment and $0.85 \mathrm{~s}$ to diagnose one subject on average. This implies that the model could be used for real-time $\mathrm{AH}$ event detection.

As Table 6 shows, our method exhibited a good sensitivity but not very good specificity compared with other studies. That is mainly because excursions in NF is not as prominent as those in nasal pressure signal during hypopnea [16] thus decrease the event detection performance. Nasal pressure signal or a combination of NF and nasal pressure signal will be taken into study in future to improve this. More importantly, the model could not only predict the severity of SAHS but could also provide time information for each $\mathrm{AH}$ event. Furthermore, compared with other methods such as convolutional neural networks, a smaller number of hyperparameters and less computation were required by our random forest based approach, and the CARTs provided better interpretability for clinical detection.

However, there were some limitations to this study. First, we did not further classify AH events into apnea events and hypopnea events. Second, the model was not tested in an online environment. We hope to confirm the usability of our method online in the future. Third, the model was not able to distinguish central and obstructive events because no ribcage or abdominal movement signals for identifying central events from obstructive events were adopted in this study. Finally, no electroencephalography was adopted in this algorithm, thus sleep and awake time were not evaluated in this study.

\section{Conclusion}

The purpose of this study was to propose a model for real-time detection of $\mathrm{AH}$ events. Based on the morphological features of NF and $\mathrm{SpO}_{2}$, the cascade of a 60 -s detector and 10-s detector could not only predict $\mathrm{AH}$ events, but could also provide time information for each $\mathrm{AH}$ event. Compared with previous research, the cascading detection model based on random forests provides better interpretation with reduced computational complexity. Therefore, it is expected to be an effective tool for SAHS diagnosis.
Compliance with ethical standards Formal consent was not required for this study, as the data had already been de-identified.

Conflict of interest The authors declare that they have no conflict of interest.

Open Access This article is distributed under the terms of the Creative Commons Attribution 4.0 International License (http:// creativecommons.org/licenses/by/4.0/), which permits unrestricted use, distribution, and reproduction in any medium, provided you give appropriate credit to the original author(s) and the source, provide a link to the Creative Commons license, and indicate if changes were made.

\section{References}

1. McNames JN, Fraser AM, Ieee I (2000) Obstructive sleep apnea classification based on spectrogram patterns in the electrocardiogram. Comput Cardiol 27:749-752. https://doi.org/10.1109/cic. 2000.898633

2. Bsoul M, Minn H, Tamil L (2011) Apnea MedAssist: real-time sleep apnea monitor using single-lead ECG. IEEE Trans Inf Technol Biomed 15(3):416-427. https://doi.org/10.1109/titb.2010. 2087386

3. Choi SH, Yoon H, Kim HS, Kim HB, Kwon HB, Oh SM, Lee YJ, Park KS (2018) Real-time apnea-hypopnea event detection during sleep by convolutional neural networks. Comput Biol Med 100: 123-131. https://doi.org/10.1016/j.compbiomed.2018.06.028

4. Gutierrez-Tobal GC, Alvarez D, del Campo F, Hornero R (2016) Utility of AdaBoost to detect sleep apnea-hypopnea syndrome from single-channel airflow. IEEE Trans Biomed Eng 63(3):636-646. https://doi.org/10.1109/tbme.2015.2467188

5. Lee H, Park J, Kim H, Lee K-J (2016) New rule-based algorithm for real-time detecting sleep apnea and hypopnea events using a nasal pressure signal. J Med Syst 40(12):282. https://doi.org/10.1007/ s10916-016-0637-8

6. Nakano H, Tanigawao T, Furukawa T, Nishima S (2007) Automatic detection of sleep-disordered breathing from a single-channel airflow record. Eur Respir J 29(4):728-736. https://doi.org/10.1183/ 09031936.00091206

7. Jung DW, Hwang SH, Cho JG, Choi BH, Baek HJ, Lee YJ, Jeong DU, Park KS (2018) Real-time automatic apneic event detection using nocturnal pulse oximetry. IEEE Trans Biomed Eng 65(3): 706-712. https://doi.org/10.1109/tbme.2017.2715405

8. Sola-Soler J, Antonio Fiz J, Morera J, Jane R (2012) Multiclass classification of subjects with sleep apnoea-hypopnoea syndrome through snoring analysis. Med Eng Phys 34(9):1213-1220. https:// doi.org/10.1016/j.medengphy.2011.12.008

9. Huang W, Guo B, Shen Y, Tang X (2017) A novel method to precisely detect apnea and hypopnea events by airflow and oximetry signals. Comput Biol Med 88:32-40. https://doi.org/10.1016/j. compbiomed.2017.06.015

10. Xie B, Minn H (2012) Real-time sleep apnea detection by classifier combination. IEEE Trans Inf Technol Biomed 16(3):469-477. https://doi.org/10.1109/TITB.2012.2188299

11. Hoa Dinh N, Wilkins BA, Cheng Q, Benjamin BA (2014) An online sleep apnea detection method based on recurrence quantification analysis. IEEE J Biomed Health Inform 18(4):1285-1293. https://doi.org/10.1109/jbhi.2013.2292928

12. Jung DW, Hwang SH, Lee YJ, Jeong D-U, Park KS (2017) Apneahypopnea index prediction using electrocardiogram acquired during the sleep-onset period. IEEE Trans Biomed Eng 64(2):295-301. https://doi.org/10.1109/tbme.2016.2554138 
13. Timus O, Dogru Bolat E (2017) k-NN-based classification of sleep apnea types using ECG. Turk J Electr Eng Comput Sci 25(4):30083023. https://doi.org/10.3906/elk-1511-99

14. St. Vincent's University Hospital University College Dublin Sleep Apnea Database (2008)

15. Goldberger AL, Amaral LAN, Glass L, Hausdorff JM, Ivanov PC, Mark RG, Mietus JE, Moody GB, Peng CK, Stanley HE (2000) PhysioBank, PhysioToolkit, and PhysioNet - components of a new research resource for complex physiologic signals. Circulation 101(23):E215-E220. https://doi.org/10.1161/01.CIR.101.23.e215

16. Berry R, Budhiraja R, Gottlieb D, Gozal D, Iber C, Kapur V, Marcus C, Mehra R, Parthasarathy S, Quan S, Redline S, Strohl K, Davidson Ward S, Tangredi M (2012) Rules for scoring respiratory events in sleep: update of the 2007 AASM manual for the scoring of sleep and associated events. Deliberations of the Sleep Apnea Definitions Task Force of the American Academy of Sleep Medicine. J Clin Sleep Med 8(5):597-619

17. Qureshi A, Ballard RD, Nelson HS (2003) Obstructive sleep apnea. J Allergy Clin Immunol 112(4):643-651. https://doi.org/10.1016/j. jaci.2003.08.031
18. Selvaraj N, Narasimhan R, Ieee (2013) Detection of sleep apnea on a per-second basis using respiratory signals. In: 2013 35th annual international conference of the IEEE engineering in medicine and biology society. IEEE engineering in medicine and biology society conference proceedings. pp 2124-2127

19. Olson LG, Ambrogetti A, Gyulay SG (1999) Prediction of sleepdisordered breathing by unattended overnight oximetry. J Sleep Res 8(1):51-55. https://doi.org/10.1046/j.1365-2869.1999.00134.x

20. Magalang UJ, Dmochowski J, Veeramachaneni S, Draw A, Mador MJ, El-Solh A, Grant BJB (2003) Prediction of the apnea-hypopnea index from overnight pulse oximetry. Chest 124(5):1694-1701. https://doi.org/10.1378/chest.124.5.1694

Publisher's note Springer Nature remains neutral with regard to jurisdictional claims in published maps and institutional affiliations. 